\title{
Some fixed point-type results for a class of extended cyclic self-mappings with a more general contractive condition
}

\author{
M De la Sen ${ }^{1 *}$ and Ravi P Agarwal ${ }^{2}$
}

\author{
* Correspondence: manuel. \\ delasen@ehu.es \\ ${ }^{1}$ Instituto de Investigacion y \\ Desarrollo de Procesos, Universidad \\ del Pais Vasco, Campus of Leioa \\ (Bizkaia), Aptdo. 644-Bilbao, 48080- \\ Bilbao, Spain \\ Full list of author information is \\ available at the end of the article
}

\begin{abstract}
This article discusses a more general contractive condition for a class of extended $(p \geq 2)$-cyclic self-mappings on the union of a finite number of subsets of a metric space which are allowed to have a finite number of successive images in the same subsets of its domain. If the space is uniformly convex and the subsets are nonempty, closed and convex, then all the iterates converge to a unique closed limiting finite sequence which contains the best proximity points of adjacent subsets and reduces to a unique fixed point if all such subsets intersect.
\end{abstract}

\section{Introduction}

A general contractive condition of rational type has been proposed in [1,2] for a partially ordered metric space. Results about the existence of a fixed point and then its uniqueness under supplementary conditions are proved in those articles. The general rational contractive condition of [3] includes as particular cases several of the existing ones [1,4-12] including Banach's principle [5] and Kannan's fixed point theorems [4,8,9,11]. The general rational contractive conditions of [1,2] are applicable only on distinct points of the considered metric spaces. In particular, the fixed point theory for Kannan's mappings is extended in [4] by the use of a non-increasing function affecting to the contractive condition and the best constant to ensure that a fixed point is also obtained. Three fixed point theorems which extended the fixed point theory for Kannan's mappings were proved in [11]. On the other hand, important attention has been paid during the last decades to the study of standard contractive and Meir-Keeler-type contractive cyclic self-mappings (see, for instance, [13-22]). More recent investigation about cyclic selfmappings is being devoted to its characterization in partially ordered spaces and to the formal extension of the contractive condition through the use of more general strictly increasing functions of the distance between adjacent subsets. In particular, the uniqueness of the best proximity points to which all the sequences of iterates converge is proven in [14] for the extension of the contractive principle for cyclic self-mappings in uniformly convex Banach spaces (then being strictly convex and reflexive [23]) if the $p$ subsets $A_{i} \subset X$ of the metric space $(X, d)$, or the Banach space $(X,\|\|)$, where the cyclic self-mappings are defined are non-empty, convex and closed. The research in [14] is centred on the case of the cyclic self-mapping being defined on the union of two subsets 
of the metric space. Those results are extended in [14] for Meir-Keeler cyclic contraction maps and, in general, for the self-mapping $T: \bigcup_{i \in \bar{p}} A_{i} \rightarrow \bigcup_{i \in \bar{p}} A_{i}$ be a $\mathrm{p}(\geq 2)$-cyclic selfmapping being defined on any number of subsets of the metric space with $\bar{p}:=\{1,2, \ldots, p\}$.

Other recent researches which have been performed in the field of cyclic maps are related to the introduction and discussion of the so-called cyclic representation of a set $M$, decomposed as the union of a set of non-empty sets as $M=\bigcup_{i=1}^{m} M_{i}$, with respect to an operator $f: M \rightarrow M$ [24]. Subsequently, cyclic representations have been used in [25] to investigate operators from $M$ to $M$ which are cyclic $\phi$-contractions, where $\phi$ : $\boldsymbol{R}_{0+} \rightarrow \boldsymbol{R}_{0+}$ is a given comparison function, $M \subset X$ and $(X, d)$ is a metric space. The above cyclic representation has also been used in [26] to prove the existence of a fixed point for a self-mapping defined on a complete metric space which satisfies a cyclic weak $\phi$-contraction. In [27], a characterization of best proximity points is studied for individual and pairs of non-self-mappings $S, T: A \rightarrow B$, where $A$ and $B$ are non-empty subsets of a metric space. In general, best proximity points do not fulfil the usual "best proximity" condition $x=S x=T x$ under this framework. However, best proximity points are proven to jointly globally optimize the mappings from $x$ to the distances $d$ $(x, T x)$ and $d(x, S x)$. Furthermore, a class of cyclic $\phi$-contractions, which contain the cyclic contraction maps as a subclass, has been proposed in [28] to investigate the convergence and existence results of best proximity points in reflexive Banach spaces completing previous related results in [14]. Also, the existence and uniqueness of best proximity points of $p(\geq 2)$-cyclic $\phi$-contractive self-mappings in reflexive Banach spaces has been investigated in [29].

In this article, it is also proven that the distance between the adjacent subsets $A_{i}, A_{i}$ ${ }_{+1} \subset X$ are identical if the $p(\geq 2)$-cyclic self-mapping is non-expansive [16]. This article is devoted to a generalization of the contractive condition of [1] for a class of extended cyclic self-mappings on any number of non-empty convex and closed subsets $A_{i} \subset X$, $i \in \bar{p}$. The combination of constants defined the contraction may be different on each of the subsets and only the product of all the constants is requested to be less than unity. On the other hand, the self-mapping can perform a number of iterations on each of the subsets before transferring its image to the next adjacent subset of the $p(\geq$ 2 ) -cyclic self-mapping. The existence of a unique closed finite limiting sequence on any sequence of iterates from any initial point in the union of the subsets is proven if $X$ is a uniformly convex Banach space and all the subsets of $X$ are non-empty, convex and closed. Such a limiting sequence is of size $q \geq p$ (with the inequality being strict if there is at least one iteration with image in the same subset as its domain) where $p$ of its elements (all of them if $q=p$ ) are best proximity points between adjacent subsets. In the case that all the subsets $A_{i} \subset X$ intersect, the above limit sequence reduces to a unique fixed point allocated within the intersection of all such subsets.

\section{Main results for non-cyclic self-mappings}

Let $(X, d)$ be a metric space for a metric $d: X \times X \rightarrow \boldsymbol{R}_{0+}$ with a self-mapping $T: X \rightarrow$ $X$ which has the following contractive condition proposed and discussed in [1]:

$$
d(T x, T y) \leq \alpha \frac{d(x, T x) d(y, T y)}{d(x, y)}+\beta d(x, y), \quad x, y(\neq x) \in X
$$


for some real constants $\alpha, \beta \in \boldsymbol{R}_{0+}$ and $\alpha+\beta<1$ where $\boldsymbol{R}_{0+}=\{r \in \boldsymbol{R}: r \geq 0\}$. A more general one involving powers of the distance is the following:

$$
d^{s(x, y)}(T x, T y) \leq \alpha \frac{d^{\sigma(x, y)}(x, T x) d^{r(x, y)}(y, T y)}{d^{\sigma(x, y)}(x, y)}+\beta d^{t(x, y)}(x, y), \quad x, y(\neq x) \in X,
$$

where $s, \sigma, r, t: X \times X \rightarrow \boldsymbol{R}_{+}=\{r \in \boldsymbol{R}: r>0\}$ are continuous and symmetric with respect to the order permutation of the arguments $x$ and $y$. It is noted that if $x=y$ then (2.1) has a sense only if $x$ is a fixed point, i.e. $x=y=T x=T y$ implies that (2.1) reduces to the inequality " $0 \leq 0$ ". The following result holds:

Theorem 2.1: Assume that the condition (2.2) holds for some symmetric continuous functions subject to $0<r(x, y) \leq s(x, y)+\ln (P-d(T x, T y))$ if $r(x, y) \neq s(x, y)$ and $0<t(x, y) \leq s(x, y)+\ln (Q-d(x, y))$ if $t(x, y) \neq s(x, y)$ for some real constants $\alpha, \beta, P, Q \geq 0$, subject to the constraint $\alpha P+\beta Q<1$. Then, $d\left(T^{n+1} x, T^{n} x\right) \rightarrow 0$ as $n \rightarrow$ $\infty ; \forall x \in X$. Furthermore, $\left\{T^{n} x\right\}_{n \in N_{0}}$ is a Cauchy sequence.

If, in addition, $(X, d)$ is complete then $T^{n} x \rightarrow z$ as $n \rightarrow \infty$, for some $z \in X$. If, furthermore, $T: X \rightarrow X$ is continuous, then $z=T z$ is the unique fixed point of $T: X \rightarrow$ $X$.

Proof: If $y=T x$, then the above given constraints on the symmetric functions become $0<r(x, x) \leq s(x, x)+\ln \left(P-d\left(T x, T^{2} x\right)\right)$ if $r(x, x) \neq s(x, x)$ and $0<t(x, x) \leq s(x, x)+\ln (Q-d(x, T x))$ if $t(x, x) \neq s(x, x)$. If $y=x=T x$, then $d\left(T^{n+1} x\right.$, $\left.T^{n} x\right) \rightarrow 0$ as $n \rightarrow \infty ; x \in X$ follows directly from (2.2) since $d^{s(x, x)}\left(T^{n+1} x, T^{n} x\right)=0$. Now, take $y=T x$ so that for any $x \neq T x$ for $x, T x \in X$ and note that the conditions 0 $<r(x, \quad x) \leq s(x, \quad x)+\ln \left(P-d\left(T x, \quad T^{2} x\right)\right) \quad$ if $\quad r(x, \quad x) \neq s(x, \quad x)$ and $0<t(x, x) \leq s(x, x)+\ln (Q-d(x, T x))$ if $t(x, x) \neq s(x, x)$ are identical to

$$
d^{r(x, x)}(x, T x) \leq P d^{s(x, x)}(x, T x) ; \quad d^{t(x, x)}(x, T x) \leq Q d^{s(x, x)}(x, T x)
$$

Thus, one gets from (2.1):

$$
d^{s(x, x)}\left(T x, T^{2} x\right) \leq \alpha d^{r(x, x)}\left(T x, T^{2} x\right)+\beta d^{t(x, x)}(x, T x) \leq \alpha P d^{s(x, x)}\left(T x, T^{2} x\right)+\beta Q d^{s(x, x)}(x, T x)
$$

so that, since $k:=\frac{\beta Q}{1-\alpha P}<1$, one gets from (2.4) proceeding by complete induction for $n \in N_{0}$

$$
0 \leftarrow d^{s(x, x)}\left(T^{n+2} x, T^{n+1} x\right) \leq \frac{\beta Q}{1-\alpha P} d^{s(x, x)}\left(T^{n+1} x, T^{n} x\right) \leq k^{n} d^{s(x, x)}(T x, x) \rightarrow 0 \text { as } n \rightarrow \infty
$$

what implies $d\left(T^{n+1 x}, T^{n} x\right) \leq k^{n / s(x, x)} d(T x, x) \rightarrow 0$ as $n \rightarrow \infty ; \forall x \in X$. Taking $n, m(\geq$ $n+2) \in N_{0}$, one can get from (2.5):

$$
d^{s(x, x)}\left(T^{m} x, T^{n+1} x\right) \leq\left(\sum_{j=n}^{m-1} k^{j}\right) d^{s(x, x)}(T x, x) \leq \frac{k^{n}}{1-k} d^{s(x, x)}(T x, x) \rightarrow 0 \text { as } n \rightarrow \infty
$$

so that

$$
d\left(T^{m} x, T^{n+1} x\right) \leq\left(\sum_{j=n}^{m-1} k^{j}\right)^{1 / s(x, x)} d(T x, x) \leq\left(\frac{k^{n}}{1-k}\right)^{1 / s(x, x)} d(T x, x) \rightarrow 0 \text { as } n, m \rightarrow \infty
$$


what proves that $\left\{T^{n} x\right\}_{n \in N_{0}}$ is a Cauchy sequence. Such a Cauchy sequence has a limit $z=\lim _{n \rightarrow \infty} T^{n} x$ in $X$ if $(X, d)$ is complete from the convergence property of Cauchy sequences to points of the space $X$. If, in addition, $T: X \rightarrow X$ is continuous then $T z=T\left(\lim _{n \rightarrow \infty} T^{n} x\right)=\lim _{n \rightarrow \infty} T^{n+1} x=z$ so that the limit of the sequence is a fixed point. The uniqueness of the fixed point is now proven (i.e. $z$ is not dependent on of $x$ ) by contradiction. Assume that there exists two distinct fixed points $y=T y$ and $z=T z$ in $X$. Then, from (2.5):

$$
d(T y, y)=d(T z, z)=0 \Rightarrow(0<d(y, z) \leq d(y, T y)+d(T y, T z)+d(T z, z)=d(T y, T z))
$$

so that

$$
0<d^{s(y, z)}(T y, T z) \leq \alpha \frac{d^{\sigma(y, z)}(y, T y) d^{r(y, z)}(z, T z)}{d^{\sigma}(y, z)}(y, z) \quad+\beta d^{t(y, z)}(y, z)=\beta d^{t(y, z)}(T y, T z) \leq \beta Q d^{s(y, z)}(T y, T z)
$$

what implies $\beta Q \geq 1$ if $d(T y, T z)=d(y, z)>0$ contradicting $d(T y, T z)=d(y, z)>0$. Thus, $y=z$ and hence the theorem.

A simpler contractive condition leads to a close result to Theorem 2.1 as follows:

Corollary 2.2: Assume that the condition (2.2) is modified as follows:

$$
d^{s}(T x, T y) \leq \alpha \frac{d^{s}(x, T x) d^{s}(y, T y)}{d^{s}(x, y)}+\beta d^{s}(x, y)
$$

for some real constants $s \in \boldsymbol{R}_{+}, \alpha, \beta \in \boldsymbol{R}_{0+}$, subject to $\alpha+\beta<1$. Then, Theorem 2.1 holds.

Proof: Taking $P=Q=1$ then (2.3)-(2.7) hold by replacing $r(x), s(x), t(x) \rightarrow s \in \boldsymbol{R}_{+}$. Thus, Theorem 2.1 holds for this particular case. Hence, the corollary. $\square$

\section{Main results for $p(\geq 2)$-cyclic self-mappings and extended $p$-cyclic self- mappings}

Let $T: \bigcup_{i \in \bar{p}} A_{i} \rightarrow \bigcup_{i \in \bar{p}} A_{i}$ be an extended $p(\geq 2)$-cyclic self-mapping where $A_{i} \equiv A_{i+k p}$ $\subset X ; \forall i \in \bar{p}:=\{1,2, \ldots ., p\}, \forall k \in N$ subject to the constraints $T\left(A_{i}\right) \subseteq A_{i} \cup A_{i+1}, T^{\ell}\left(A_{i}\right)$ $\subseteq A_{i+1} ; \forall \ell \in \overline{j_{i}-1}$ and $T^{j_{i}}\left(A_{i}\right) \subseteq A_{i+1}$ for some finite integers $j_{i} \geq 1 ; \forall i \in \bar{p}$ (this implies that $q:=\sum_{i=1}^{p} j_{i} \geq p$ with equality standing if and only if $j_{i} \geq 1 ; \forall i \in \bar{p}$, i.e. if the cyclic mapping is of standard type) with $T^{k}=T \circ T^{k-1}$ and $T^{0} \equiv i d$. It is noted that the extended $p(\geq 2)$-cyclic self-mapping $T: \bigcup_{i \in \bar{p}} A_{i} \rightarrow \bigcup_{i \in \bar{p}} A_{i}$ is characterized by the $p$ tuple of integers $\left(j_{i}: i \in \bar{p}\right)$, where $\sum_{i=1}^{p} j_{i}=q \geq p$ and if, in particular, $j_{i}=1 ; \forall i \in \bar{p}$ then $T: \bigcup_{i \in \bar{p}} A_{i} \rightarrow \bigcup_{i \in \bar{p}} A_{i}$ is the standard $p$-cyclic self-mapping. It is also noted that the self-mappings $T^{j_{i}}: \bigcup_{i \in \bar{p}} A_{i} \rightarrow \bigcup_{i \in \bar{p}} A_{i}$ and the composed mappings $T^{q+j_{i}}: \bigcup_{i \in \bar{p}} A_{i} \rightarrow \bigcup_{i \in \bar{p}} A_{i}$ satisfying the extended inclusion constraint $T\left(A_{i}\right) \subseteq A_{i} \cup A_{i+1}$, subject to $T^{\ell}\left(A_{i}\right) \subseteq A_{i}, T^{j_{i}}\left(A_{i}\right) \subseteq A_{i+1} ; \forall \ell \in \overline{j_{i}-1} ; \forall i \in \bar{p}$, are not $q$-cyclic self-mappings [13-17], except if $q=p$, since $T^{q+j_{\ell}}\left(A_{i}\right) \subseteq A_{i+\ell}$ fails for $i,(\ell \neq i) \in \bar{p}$ unless $j_{\ell} \geq j_{i}$. The contractive condition (2.1) becomes modified as follows:

$$
d^{s(x, y)}(T x, T y) \leq \alpha_{i} \frac{d^{\sigma(x, y)}(x, T x) d^{r(x, y)}(y, T y)}{d^{\sigma(x, y)}(x, y)}+\beta_{i} t^{t(x, y)}(x, y)+\gamma_{i} D^{s(x, y)}
$$


for $x, y \in A_{i} \cup A_{i+1}, T x \in A_{i} \cup A_{i+1}, T y \in A_{i+1} \cup A_{i+2}$ and some real constants $\gamma_{i} \in$ $\boldsymbol{R}_{0+}$ while $T x, T y$ are not both in the same subset $A_{j}$ for $j=i, i+1, i+2$ for any $i \in \bar{p}$, and

$$
d^{s(x, y)}(T x, T y) \leq \alpha_{i} \frac{d^{\sigma(x, y)}(x, T x) d^{r(x, y)}(y, T y)}{d^{\sigma(x, y)}(x, y)}+\beta_{i} d^{t(x, y)}(x, y) \text { if } x, y \in A_{i}, T x, T y \in A_{i}
$$

or if $x, y \in A_{i+1}, T x, T y \in A_{i+1}$ for any $i \in \bar{p}$, where $D:=\operatorname{dist}\left(A_{i}, A_{i+1}\right)$ being zero if $\forall i \in \bar{p} ; \forall i \in \bar{p}$. Fix $y=T x$ then, one can get from (3.2) for $x \in A_{i}$ :

$$
\left(1-\alpha_{i} P_{i}\right) d^{s(x, x)}\left(T x, T^{2} x\right) \leq \beta_{i} d^{t(x, x)}(x, T x)+\left(1-\gamma_{i}\right) D \leq \beta_{i} Q_{i} d^{s(x, x)}(x, T x)+\gamma_{i} D^{s(x, x)}
$$

if $T x \in A_{i+1}, \forall i \in \bar{p}$, and

$$
d^{s(x, x)}\left(T x, T^{2} x\right) \leq \alpha_{i} d^{r(x, x)}\left(T x, T^{2} x\right)+\beta_{i} d^{t(x, x)}(x, T x) \leq \alpha_{i} P_{i} d^{s(x, x)}\left(T x, T^{2} x\right)+\beta_{i} Q_{i} d^{s(x, x)}(x, T x)
$$

if $x, T x \in A_{i}$ provided that the following upper-bounding conditions hold:

$$
\begin{aligned}
& d^{r(x, x)}(x, T x) \leq P_{i} d^{s(x, x)}(x, T x) ; \quad d^{t(x, x)}(x, T x) \leq Q_{i} d^{s(x, x)}(x, T x) \\
& \alpha_{i}, \beta_{i}, P_{i}, Q_{i} \geq 0
\end{aligned}
$$

Thus, the following technical result holds which does not require completeness of the metric space, uniform convexity assumption on some associated Banach space or particular properties of the non-empty subsets $A_{i} ; \forall i \in \bar{p}$. The result will be then used to obtain the property of convergence of the sequences of iterates to best proximity points allocated in the various subsets.

Theorem 3.1: Let $(X, d)$ a metric space and $A_{i} \equiv A_{i+k p} \subset X ; \forall i \in \bar{p}$. Assume that $T: \bigcup_{i \in \bar{p}} A_{i} \rightarrow \bigcup_{i \in \bar{p}} A_{i}$ is an extended (p $\geq 2$ ) $p$-cyclic map, subject to the extended contractive condition (3.1), with $T\left(A_{i}\right) \subseteq A_{i} \cup A_{i+1}, T^{\ell}\left(A_{i}\right) \subseteq A_{i+1} ; \forall \ell \in \overline{j_{i}-1}$ and $T^{j_{i}}\left(A_{i}\right) \subseteq A_{i+1}$ for some finite integers $j_{i} \geq 1$ and $q:=\sum_{i=1}^{p} j_{i} \geq p ; \forall i \in \bar{p}$. Define $k_{i}:=\left(\frac{\beta_{i} Q_{i}}{1-\alpha_{i} P_{i}}\right)^{j_{i}}$, subject to $k:=\left[\prod_{i=1}^{p} k_{i}\right]<1$, and $\gamma_{i}=1-k_{i} ; \forall i \in \bar{p}$. Assume also that $\sigma(x, x)>0, s(x, x)>0,0<r(x, x) \leq s(x, x)+\ln \left(P-d\left(T x, T^{2} x\right)\right)$ if $r(x, x) \neq s(x, x)$ and $0<t(x, x) \leq s(x, x)+\ln (Q-d(x, T x))$ if $t(x, x) \neq s(x, x) ; \forall x \in \bigcup_{i \in \bar{p}} A_{i}$. Then, the following properties hold:

(i)

$$
\begin{aligned}
& \lim _{n \rightarrow \infty} d^{s(x, x)}\left(T^{n q+j_{i}} x, T^{n q} x\right)=D^{s(x, x)} ; \quad \lim _{n \rightarrow \infty} d\left(T^{n q+j_{i}} x, T^{n q} x\right)=D \forall x \in A_{i}, \forall i \in \bar{p} ; \text { (3.6a) } \\
& \lim _{n \rightarrow \infty} d\left(T^{n q+j_{i}+j_{i+1}} x, T^{n q+j_{i}} x\right)=D ; \quad \lim _{n \rightarrow \infty} d\left(T^{n q+\sum_{\ell=i}^{m} j_{\ell}} x, T^{n q+j_{i}} x\right)=D \\
& \lim _{n \rightarrow \infty} d\left(T^{n q+\sum_{\ell=i}^{m} j_{\ell}} x, T^{n q+\sum_{\ell=i}^{m^{\prime}} j_{\ell}} x\right)=D ; \forall x \in A_{i} \forall i \in \bar{p},
\end{aligned}
$$

with $i \leq m^{\prime}<m<p+i, j_{p+i}=j_{p} ; \forall i \in \bar{p}$, and similarly:

$$
\limsup _{n \rightarrow \infty} d^{s(x, x)}\left(T^{n q+\ell} x, T^{n q} x\right) \leq\left(\frac{\beta_{i} Q_{i}}{1-\alpha_{i} P_{i}}\right)^{\ell} D^{s(x, x)} ; \forall x \in A_{i} ; \forall \ell \in \overline{j_{i}-1}, \forall i \in \bar{p}(3
$$




$$
\limsup _{n \rightarrow \infty} d\left(T^{n q+\ell} x, T^{n q} x\right) \leq\left(\frac{\beta_{i} Q_{i}}{1-\alpha_{i} P_{i}}\right)^{\ell / s(x, x)} D ; \forall x \in A_{i} ; \forall \ell \in \overline{j_{i}-1}, \forall i \in \bar{p}
$$

(ii)

$$
\begin{aligned}
& \lim _{n \rightarrow \infty} d^{s(x, x)}\left(T^{(n+m) q+j_{i}} x, T^{n q} x\right)=D^{s(x, x)} ; \lim _{n \rightarrow \infty} d\left(T^{(n+m) q+j_{i}} x, T^{n q} x\right)=D ; \forall x \in A_{i}, \forall m \in N, \forall i \in \bar{p} \\
& \limsup _{n \rightarrow \infty} d^{s(x, x)}\left(T^{(n+m) q+\ell} x, T^{n q} x\right) \leq\left(\frac{\beta_{i} Q_{i}}{1-\alpha_{i} P_{i}}\right)^{\ell} D^{s(x, x)} ; \forall x \in A_{i} ; \forall \ell \in \overline{j_{i}-1}, \forall m \in N, \forall i \in \bar{p} \\
& \limsup _{n \rightarrow \infty} d\left(T^{(n+m) q+\ell} x, T^{n q} x\right) \leq\left(\frac{\beta_{i} Q_{i}}{1-\alpha_{i} P_{i}}\right)^{\ell / s(x, x)} D ; \forall x \in A_{i} ; \forall \ell \in \overline{j_{i}-1}, \forall m \in N, \forall i \in \bar{p}
\end{aligned}
$$

Proof: The proof of Property (i) follows from the following inequalities which follow by recursion from (3.3) to (3.5):

$$
\begin{aligned}
& d^{s(x, x)}\left(T^{\ell+1} x, T^{\ell} x\right) \leq\left(\frac{\beta_{i} Q_{i}}{1-\alpha_{i} P_{i}}\right)^{\ell} d^{s(x, x)}(x, T x) \\
& d^{s(x, x)}\left(T^{j_{i}} x, T^{j_{i}-1} x\right) \leq k_{i} d^{s(x, x)}(x, T x)+\left(1-k_{i}\right) D^{s(x, x)} \\
& d^{s(x, x)}\left(T^{q+1} x, T^{q} x\right) \leq k d^{s(x, x)}(x, T x)+(1-k) D
\end{aligned}
$$

$\forall x \in A_{i}, T^{\ell} x \in A_{i}, T^{j_{i}} x \in A_{i+1} ; \forall \ell \in \overline{j_{i}-1}, \forall i \in \bar{p}$ since $q:=\sum_{i=1}^{p} j_{i} \geq p$. One can get from (3.12) and (3.14) and, respectively, from (3.13) to (3.14):

$$
\begin{aligned}
& d^{s(x, x)}\left(T^{q+\ell} x, T^{q} x\right) \leq\left(\frac{\beta_{i} Q_{i}}{1-\alpha_{i} P_{i}}\right)^{\ell} d^{s(x, x)}\left(T^{q+1} x, T^{q} x\right) \leq\left(\frac{\beta_{i} Q_{i}}{1-\alpha_{i} P_{i}}\right)^{\ell}\left[k d^{s(x, x)}(x, T x)+(1-k) D\right] \\
& d^{s(x, x)}\left(T^{q+j_{i}} x, T^{q} x\right) \leq k_{i} d^{s(x, x)}\left(T^{q+1} x, T^{q} x\right)+\left(1-k_{i}\right) D^{s(x, x)} \\
& \leq k_{i}\left[k d^{s(x, x)}(x, T x)+(1-k) D\right]+\left(1-k_{i}\right) D^{s(x, x)}
\end{aligned}
$$

Proceeding recursively with (3.14) for $n \in N$, one can get:

$$
d^{s(x, x)}\left(T^{n q+\ell} x, T^{n q} x\right) \leq\left(\frac{\beta_{i} Q_{i}}{1-\alpha_{i} P_{i}}\right)^{\ell}\left[k^{n} d^{s(x, x)}(x, T x)+\left(1-k^{n}\right) D^{s(x, x)}\right] ; x, T^{\ell} x \in A_{i}
$$

for $\ell \in \overline{j_{i}-1}$, and

$$
D^{s(x, x)} \leq d^{s(x, x)}\left(T^{n q+j_{i}} x, T^{n q} x\right) \leq k_{i}\left[k^{n} d^{s(x, x)}(x, T x)+\left(1-k^{n}\right) D^{s(x, x)}\right]+\left(1-k_{i}\right) D^{s(x, x)}
$$

; $\forall x \in A_{\mathrm{i}} ; T^{n q} x \in A_{i} ; T^{n q+j_{i}} x \in A_{i+1} ; \forall i \in \bar{p}$. One can get (3.6a) from (3.18) and (3.7)(3.8) from (3.17), respectively, since $k<1$ by taking limits as $n \rightarrow \infty$. Equations 3.6b and 3.6c follow directly from (3.6a) as follows:

$$
\begin{aligned}
\qquad \lim _{n \rightarrow \infty} \sup d\left(T^{n q+j_{i}+j_{i+1}} x, T^{n q+j_{i}} x\right) & \leq \liminf _{n \rightarrow \infty}\left(k_{i} d\left(T^{n q+j_{i}} x, T^{n q} x\right)+\left(1-k_{i}\right) D\right) & \\
& =\lim _{n \rightarrow \infty}\left(k_{i} d\left(T^{n q+j_{i}} x, T^{n q} x\right)+\left(1-k_{i}\right) D\right)=D & \\
\text { with } \quad T^{n q+j_{i}+j_{i+1}} x \in A_{i+2} ; & \forall i \in \bar{p} \quad \text { since } \quad T^{n q+j_{i}} x \in A_{i+1} . & \text { Then, } \\
\exists \lim _{n \rightarrow \infty} d\left(T^{n q+j_{i}+j_{i+1}} x, T^{n q+j_{i}} x\right)=D . & &
\end{aligned}
$$


Proceeding recursively:

$$
\begin{aligned}
& \lim _{n \rightarrow \infty} \sup d\left(T^{n q+\sum_{\ell=i}^{m} j_{\ell}} x, T^{n q+j_{i}} x\right) \leq \liminf _{n \rightarrow \infty}\left(\prod_{\ell=i_{1}}^{m-1}\left[k_{\ell}\right] d\left(T^{n q+j_{i}} x, T^{n q} x\right)\right. \\
& \left.+\left(1-\prod_{\ell=i_{1}}^{m-1}\left[k_{\ell}\right]_{i}\right) D\right)=\lim _{n \rightarrow \infty}\left(k_{i} d\left(T^{n q+j_{i}} x, T^{n q} x\right)+\left(1-k_{i}\right) D\right)=D
\end{aligned}
$$

with $i \quad<m(\in \quad N) \quad<p+i, \quad j_{p+i}=j_{p}, \quad T^{n q+\sum_{\ell=i}^{m} j_{\ell}} x \in A_{i+m+1} ; \quad \forall i \in \bar{p}$ so that $\exists \lim _{n \rightarrow \infty} d\left(T^{n q+\sum_{\ell=i}^{m} j_{\ell}} x, T^{n q+j_{i}} x\right)=D$. In the same way, one can get:

$$
\begin{aligned}
& \lim _{n \rightarrow \infty} \sup d\left(T^{n q+\sum_{\ell=i}^{m} j_{\ell}} x, T^{n q+\sum_{\ell=i}^{m-m^{\prime}} j_{\ell}} x\right) \\
& \leq \liminf _{n \rightarrow \infty}\left(\prod_{\ell=m-m^{\prime}}^{m-1}\left[k_{\ell}\right] d\left(T^{n q+\sum_{\ell=i}^{m-m^{\prime}} j_{\ell}} x, T^{n q} x\right)+\left(1-\prod_{\ell=m-m^{\prime}}^{m-1}\left[k_{\ell}\right]\right) D\right) \\
& =\lim _{n \rightarrow \infty}\left(k_{i} d\left(T^{n q+j_{i}} x, T^{n q} x\right)+\left(1-k_{i}\right) D\right)=D
\end{aligned}
$$

with $i \leq m^{\prime}+\mathrm{i} \leq(\in \boldsymbol{N}) \leq p+i, j_{p+i}=j_{p}, T^{n q+\sum_{\ell=i}^{m-m_{1}} j_{\ell} \in A_{i+m-m_{1}+1}} ; \forall i \in \bar{p}$. Then, $\exists \lim _{n \rightarrow \infty} d\left(T^{n q+\sum_{\ell=i}^{m} j_{\ell}} x, T^{n q+\sum_{\ell=i}^{m-m^{\prime}} j_{\ell}} x\right)=D$. Property (i) has been proven. Now, note from (3.16), (3.15) and (3.18) that

$$
\begin{aligned}
& D^{s(x, x)} \leq d^{s(x, x)}\left(T^{(n+m) q+\ell} x, T^{n q} x\right) \leq\left(\frac{\beta_{i} Q_{i}}{1-\alpha_{i} P_{i}}\right)^{\ell} d^{s(x, x)}\left(T^{(n+m) q} x, T^{n q} x\right) \\
& \leq k_{i}\left[k^{n+m} d^{s(x, x)}(x, T x)+\left(1-k^{n+m}\right) D^{s(x, x)}\right]+\left(1-k_{i}\right) D^{s(x, x)} \\
& D^{s(x, x)} \leq d^{s(x, x)}\left(T^{(n+m) q+j_{i}} x, T^{(n+m) q} x\right) \leq k_{i} d^{s(x, x)}\left(T^{(n+m) q} x, T^{n q} x\right) \\
& \leq k_{i}\left[k^{n+m} d^{s(x, x)}(x, T x)+\left(1-k^{n+m}\right) D^{s(x, x)}\right]+\left(1-k_{i}\right) D^{s(x, x)}
\end{aligned}
$$

$; \forall x \in A_{i}, T^{\ell} x \in A_{i}, T^{j_{i}} x \in A_{i+1} ; \forall \ell \in \overline{j_{i}-1}, \forall m \in N, \forall i \in \bar{p}$. Hence, Property (ii). $\square$

Remark 3.2. It is noted that if $A_{i} \cap A_{\ell}=\emptyset$ and $x \in A_{j}$ for some $i, \ell(\neq i) \in \bar{p}$ and $j_{\ell}<j_{i}$ then $d\left(T^{(n+1) q+\ell} x, T^{n q+\ell} x\right) \rightarrow D$ as $n \rightarrow \infty$ for all $\ell<j_{i}$.

The following result is concerned with the proved property that distances of iterates obtained through the composed self-mapping $T^{q}: \bigcup_{i \in \bar{p}} A_{i} \rightarrow \bigcup_{i \in \bar{p}} A_{i}$ starting from a point $x$ in any of the subsets, and located within two distinct of such subsets for all the iteration steps, asymptotically converge to the distance $D$ between such subsets in uniformly convex Banach spaces, with at least one of them being convex. It is also obtained a convergence property of the iterates of the composed self-mapping $T^{q}: \bigcup_{i \in \bar{p}} A_{i} \rightarrow \bigcup_{i \in \bar{p}} A_{i}$ to limit points within each of the subsets.

Lemma 3.3: Let $(X,\|\|)$ be a uniformly convex Banach space endowed with the norm \|\| and let $d: \mathrm{X} \times \mathrm{X} \rightarrow \boldsymbol{R}_{0+}$ be a metric induced by such a norm \|\| so that $(X$, $d)$ is a complete metric space. Assume that the non-empty subsets $A_{i}$ of $X$ and the extended $p(\geq 2)$-cyclic self-mapping $T: \bigcup_{i \in \bar{p}} A_{i} \rightarrow \bigcup_{i \in \bar{p}} A_{i}$ fulfil the constraints of Theorem 3.1 and, furthermore, one subset is closed and another one is convex and closed in each pair $\left(A_{i}, A_{i+1}\right)$ of adjacent subsets, $\forall i \in \bar{p}$. Then, the following properties hold:

(i)

$$
\lim _{n \rightarrow \infty} d\left(T^{\left(n+n^{\prime}\right) q+j_{i}} x, T^{n q+j_{i}} x\right)=\lim _{n \rightarrow \infty} d\left(T^{\left(n+n^{\prime}\right) q+\sum_{\ell=i}^{m} j_{\ell}} x, T^{n q+\sum_{\ell=i}^{m} j_{\ell}} x\right)=0
$$




$$
\lim _{n \rightarrow \infty} d\left(T^{\left(n+n^{\prime}\right) q+j_{i}} x, T^{n q} x\right)=\lim _{n \rightarrow \infty} d\left(T^{\left(n+n^{\prime}\right) q+\sum_{\ell=i}^{m} j_{\ell}} x, T^{n q+\sum_{\ell=i}^{m^{\prime}} j_{\ell}} x\right)=D ; \forall x \in A_{i}, \forall i \in \bar{p}
$$

; $\forall x \in A_{i}, i \leq m^{\prime}<m<p+i, j_{p+i}=j_{p}, m, m^{\prime} \in \bar{p}, \forall n^{\prime} \in N, \forall i \in \bar{p}$. Furthermore, if $A_{i+1}$ is convex, then $T^{n q+j_{i}} x \rightarrow z_{i+1} \in A_{i+1} \quad$ as $\mathrm{n} \quad \rightarrow \quad \infty$. Also, $T^{n q+\sum_{\ell=1}^{m} j_{\ell}} x \rightarrow z_{i+m+1}=T^{\sum_{\ell=1}^{m} j_{\ell}} z_{i}\left(\in A_{i+m+1}\right)$ as $\mathrm{n} \rightarrow \infty$ with $z_{i+m+1} \equiv z_{i+m+1-p}, A_{i+m+1} \equiv A_{i}$ $+m+1-p$ if $m>p+1-i$. Furthermore, if all the subsets $A_{i}(i \in \bar{p})$ are closed and convex, then $T^{q n} x \rightarrow z\left(\in \bigcap_{i \in \bar{p}} A_{i}\right)=T^{q} z$ as $\mathrm{n} \rightarrow \infty$ if $D=0$, that is if $\bigcap_{i \in \bar{p}} A_{i} \neq \emptyset$, so that $z \in \bigcap_{i \in \bar{p}} A_{i}$ is the unique fixed point of $T^{q}: \bigcup_{i \in \bar{p}} A_{i} \rightarrow \bigcup_{i \in \bar{p}} A_{i}$ in $\bigcap_{i \in \bar{p}} A_{i}$

(ii) If $A_{i}$ or $A_{i+m+1}$ is convex then

$$
\lim _{n \rightarrow \infty} d\left(T^{\left(n+n^{\prime}\right) q+\sum_{\ell=i}^{m} j_{\ell}} x, T^{n q+\sum_{\ell=i}^{m} j_{\ell}} x\right)=0
$$

; $\forall x \in A_{i}, i \leq m<p+i, j_{p+i}=j_{p}, \forall n^{\prime} \in N_{0}:=N \cup\{0\}, \forall i \in \bar{p}$

Proof: Note from (3.6a) that

$$
\left(\left[d\left(T^{n q+j_{i}} x, T^{n q} x\right) \rightarrow D\right] \wedge\left[d\left(T^{\left(n+n^{\prime}\right) q+j_{i}} x, T^{n q} x\right) \rightarrow D\right]\right) \Rightarrow\left(d\left(T^{\left(n+n^{\prime}\right) q+j_{i}} x, T^{n q+j_{i}} x\right) \rightarrow 0\right) \text { as } n \rightarrow \infty
$$

; $\forall x \in A_{i}, j_{p+\mathrm{i}}=j_{p}, \forall n^{\prime} \in N, \forall i \in \bar{p}$ with $T^{n q} x \in A_{i}, T^{n q+j_{i}} x, T^{\left(n+n^{\prime}\right) q+j_{i}} x \in A_{i+1}$ with $A_{i}$ ${ }_{+1} \equiv A_{i+1-p}$ if $i>p-1$, since $(X,\|\|)$ is a uniformly convex Banach space, $d: \mathrm{X} \times \mathrm{X} \rightarrow$ $\boldsymbol{R}_{0+}$ be a metric induced by the norm \|\| , so that $(X, d)$ is a complete metric space, and $A_{i}$ and $A_{i+1}$ are non-empty closed subsets of $X$ and at least one of them is convex (see Lemma 3.8 of [13]). Then $\lim _{n \rightarrow \infty} d\left(T^{\left(n+n^{\prime}\right) q+j_{i}} x, T^{n q+j_{i}} x\right)=0$. On the other hand, $\lim _{n \rightarrow \infty} d\left(T^{\left(n+n^{\prime}\right) q+\sum_{\ell=i}^{m} j_{\ell}} x, T^{n q+\sum_{\ell=i}^{m} j_{\ell}} x\right)=0$ is proven by replacing (3.26a) by

$$
\begin{aligned}
& \left(\left[d\left(T^{n q+\sum_{\ell=i}^{m} j_{\ell}} x, T^{n q} x\right) \rightarrow D\right] \wedge\left[d\left(T^{\left(n+n^{\prime}\right) q+\sum_{\ell=i}^{m} j_{\ell}} x, T^{n q} x\right) \rightarrow D\right]\right) \\
& \Rightarrow\left(d\left(T^{\left(n+n^{\prime}\right) q+\sum_{\ell=i}^{m} j_{\ell}} x, T^{n q+\sum_{\ell=i}^{m} j_{\ell}} x\right) \rightarrow 0\right) \text { as } n \rightarrow \infty
\end{aligned}
$$

with $i \leq m<p+i, \forall n^{\prime} \in N$. Thus, $x \in A_{i}$ implies $T^{n q} x \in A_{i}$, $T^{n q+\sum_{\ell=i}^{m} j_{\ell}} x, T^{\left(n+n^{\prime}\right) q+\sum_{\ell=i}^{m} j_{\ell}} x \in A_{i+m+1}$. The identities (3.24a) have been proven. To prove (3.24b), note from Equation 3.24a of Property (i) and the triangle inequality that the following holds:

$$
\begin{aligned}
& \lim _{n \rightarrow \infty} d\left(T^{\left(n+n^{\prime}\right) q+j_{i}} x, T^{n q} x\right) \leq \lim _{n \rightarrow \infty} d\left(T^{n q+j_{i}} x, T^{n q} x\right) \\
& +\lim _{n \rightarrow \infty} d\left(T^{\left(n+n^{\prime}\right) q+j_{i}} x, T^{n q+j_{i}} x\right)=\lim _{n \rightarrow \infty} d\left(T^{n q+j_{i}} x, T^{n q} x\right) \\
& =D \\
& \lim _{n \rightarrow \infty} d\left(T^{\left(n+n^{\prime}\right) q+\sum_{\ell=i}^{m} j_{\ell}} x, T^{n q+\sum_{\ell=i}^{m^{\prime}} j_{\ell}} x\right) \leq \lim _{n \rightarrow \infty} d\left(T^{n q+\sum_{\ell=i}^{m} j_{\ell}} x, T^{n q+\sum_{\ell=i}^{m^{\prime}} j_{\ell}} x\right) \\
& +\lim _{n \rightarrow \infty} d\left(T^{n q+\sum_{\ell=i}^{m^{\prime}} j_{\ell}} x, T^{\left(n+n^{\prime}\right) q+\sum_{\ell=i}^{m} j_{\ell}} x\right)=\lim _{n \rightarrow \infty} d\left(T^{n q+\sum_{\ell=i}^{m^{\prime}} j_{\ell}} x, T^{\left(n+n^{\prime}\right) q+\sum_{\ell=i}^{m} j_{\ell}} x\right) \\
& =D
\end{aligned}
$$

; $\forall x \in A_{i}, \quad i \leq m^{\prime} \quad<m<p+i, \quad j_{p+i}=j_{p}, \quad \forall m, m^{\prime} \in \bar{p}, \forall n^{\prime} \in N, \forall i \in \bar{p}$. $\exists \lim _{n \rightarrow \infty} d\left(T^{\left(n+n^{\prime}\right) q+\sum_{\ell=i}^{m} j_{\ell}} x, T^{n q+\sum_{\ell=i}^{m^{\prime}} j_{\ell}} x\right)=\lim _{n \rightarrow \infty} d\left(T^{\left(n+n^{\prime}\right) q+j_{i}} x, T^{n q} x\right)=D$. On the other hand, note that $\left\{T^{n q+j_{i}} x\right\}_{n \in N}$ is a Cauchy sequence since 
$\lim _{n \rightarrow \infty n^{\prime} \rightarrow \infty} d\left(T^{\left(n+n^{\prime}\right) q+\sum_{\ell=i}^{m} j_{\ell}} x, T^{n q+\sum_{\ell=i}^{m} j_{\ell}} x\right)=0$ from (3.24a) which then has a limit in $X$ which is also in the closed and convex subset $A_{i+1}$ of $X$. The proof of $T^{n q+\sum_{\ell=i}^{m} j_{\ell} x \rightarrow z_{i+m+1}}\left(\in A_{i+m+1}\right)$ with $A_{i+m+1} \neq A_{i}$, since $m<p+i$, as $\mathrm{n} \rightarrow \infty$ follows from similar arguments since one of the subsets in each adjacent pair of subsets is convex and both of them are closed by assumption so that $T^{n q+\sum_{\ell=i}^{m} j_{\ell}} x=T^{\sum_{\ell=i+1}^{m} j_{\ell}}\left(T^{n q+j_{i}} x\right) \rightarrow z_{i+m+1}=T^{\sum_{\ell=i+1}^{m} j_{\ell}} z_{i+1}$ as $\mathrm{n} \rightarrow \infty ; \forall x \in A_{i}, \forall i \in \bar{p}$. Finally, if the subsets intersect and are closed and convex then the composed selfmapping $T^{q}: \bigcup_{i \in \bar{p}} A_{i} \rightarrow \bigcup_{i \in \bar{p}} A_{i}$ is contractive, then continuous everywhere in its definition domain, so that it converges to a unique fixed point in the non-empty, closed and convex set $\bigcap_{i \in \bar{p}} A_{i}$. Hence, Property (i).

To prove Property (ii), note from (3.6d) with $m=i$ and $m_{1}=0$ that

$$
\begin{aligned}
& \left(\left[d\left(T^{\left(n+n^{\prime}\right) q+\sum_{\ell=i}^{m} j_{\ell}} x, T^{n q} x\right) \rightarrow D\right] \wedge\left[d\left(T^{n q+\sum_{\ell=i}^{m} j_{\ell}} x, T^{n q} x\right) \rightarrow D\right]\right) \\
& \Rightarrow\left(d\left(T^{(n+m) q+j_{i}} x, T^{n q+j_{i}} x\right) \rightarrow 0\right) \text { as } n \rightarrow \infty
\end{aligned}
$$

; $\forall x \in A_{i}, i \leq m<p+i, j_{p+i}=j_{p}, \forall n^{\prime} \in N ; \forall i \in \bar{p}$ with $T^{n q} x \in A_{i}$, $\forall i \in \bar{p} ; \forall i \in \bar{p}$ with $A_{i+m+1} \equiv A_{i+m+1-p}$ if $m>p-i-1 ; \forall i \in \bar{p}$, since $(X,\|\|)$ is a uniformly convex Banach space (and then $(X, d)$ is a complete metric space) and $A_{i}$ and $A_{i+m+1}$ are non-empty closed subsets of $X$ and $A_{i}$ or $A_{i+m+1}$ is convex. Then, (3.25) follows in the same way as Property (i). $\square$

The following result concerning to convergence of the iterates to closed finite sequences-eventually to unique fixed points if all the subsets intersect-is supported by Theorem 3.1 and Lemma 3.3.

Theorem 3.4: Let $A_{i}$ be non-empty closed and convex subsets of a uniformly convex Banach space $(X,\|\|) ; \forall i \in \bar{p}$. Assume that $T: \bigcup_{i \in \bar{p}} A_{i} \rightarrow \bigcup_{i \in \bar{p}} A_{i}$ is an extended $(p \geq$ 2) -cyclic map, subject to the extended contractive condition (3.1), with $T\left(A_{i}\right) \subseteq A_{i} \cup$ $A_{i+1}, T^{\ell}\left(A_{i}\right) \subseteq A_{i+1} ; \forall \ell \in \overline{j_{i}-1}$ and $T^{j_{i}}\left(A_{i}\right) \subseteq A_{i+1}$ for some finite integers $j_{i} \geq 1, \forall i \in \bar{p}$ and $q:=\sum_{i=1}^{p} j_{i} \geq p$. Then, the following properties hold:

(i) $T^{q n} x \rightarrow z_{i} \in A_{i}, \forall x \in A_{i}$ as $n \rightarrow \infty$ and there is a $q$-tuple:

$$
\begin{aligned}
& \hat{z}_{i}:=\left(T z_{i}=T^{q+1} z_{i}, \ldots, \omega_{i+1}=T^{j_{i}} z_{i}, T^{j_{i}+1} z_{i}, \ldots, \omega_{i+2}=T^{j_{i}+j_{i+1}} z_{i},\right. \\
& \left.T^{j_{i}+j_{i+1}+1} z_{i}, \ldots, \omega_{i+p}=\omega_{i}=z_{i}=T^{q} z_{i}\right)
\end{aligned}
$$

; $\forall i \in \bar{p}$ which is the unique limit sequence of limit points of any $q$-tuple of sequences:

$$
\hat{x}_{q n}:=\left(T^{q n+1} x, \ldots, T^{q n+j_{i}} x, T^{q n+j_{i}+1} x, \ldots, T^{q n+j_{i}+j_{i+1}} x, T^{q n+j_{i}+j_{i+1}+1} x, \ldots, T^{(q+1) n} x\right)
$$

; $\forall x \in A_{i}$, where $T^{k} z_{i} \in A_{i} ; \forall \in k \in \overline{j_{i}-1} \cup\{0\} ; \forall i \in \bar{p}, \omega_{i+\ell} \in A_{i+\ell}$ is the unique best proximity point in $A_{i+\ell} ; \forall \ell \in \bar{p}$ such that $D=\operatorname{dist}\left(A_{i}, A_{i+1}\right)=d\left(\omega_{i}, \omega_{i+1}\right) ; \forall i \in \bar{p}$.

(ii) Assume that $\bigcap_{i \in \bar{p}} A_{i} \neq \emptyset$. Then, the self-mapping $T: \bigcup_{i \in \bar{p}} A_{i} \rightarrow \bigcup_{i \in \bar{p}} A_{i}$ has a unique fixed point $z \in \bigcap_{i \in \bar{p}} A_{i}$. Then, any $q$-tuple of sequences (3.31) converges to a unique limit $q$-tuple (3.30) of the form $\hat{z}$ : $=(z, \ldots ., z)$ for any $x \in \bigcup_{i \in \bar{p}} A_{i}$ and for any $i \in \bar{p}$. 
Proof: To keep a coherent treatment with the previous part of the manuscript and, since $(X,\|\|)$ is a Banach space with norm \|\| , we can use a norm-induced metric $d$ : $X \times X \rightarrow \boldsymbol{R}_{0+}$ which is equivalent to any other metric and then apply Theorem 3.1 to the metric space $(X, d)$ which is complete since $(X,\|\|)$ is a Banach space. Assume the following cases:

(A) $\mathrm{D}=0$ so that $A_{i} \cap A_{j} \neq \emptyset$ for $i, j(\neq i) \in \bar{p}$; i.e. all the subsets have a non-empty intersection. Then, $d\left(T^{n q+j_{i}} x, T^{n q} x\right) \rightarrow 0, d\left(T^{(n+1) q} x, T^{n q} x\right) \rightarrow 0$ and $d\left(T^{n q+\ell} x, T^{n q} x\right) \rightarrow$ $0 ; \forall x \in A_{i} ; \forall \ell \in\left\{\sum_{k=i}^{m+i-1} j_{k}: m \in \bar{p}\right\}, \forall i \in \bar{p}$ as $n \rightarrow \infty$ from Theorem 3.1, Equations 3.6 and 3.8, with $A_{j} \equiv A_{j-p}$ for $2 p \geq j>p$. Thus, $T^{q n} x\left(\in A_{i}\right) \rightarrow z$, since $\left\{T^{q n} x\right\}_{n} \in N$ is a Cauchy sequence (and also $T^{q+\ell} x\left(\in A_{i+\ell}\right) \rightarrow z$ ) for some $z \in \bigcap_{i \in \bar{p}} A_{i}$, since $\bigcap_{i \in \bar{p}} A_{i}$ is nonempty, convex and closed from Banach contraction principle since $k<1$. Since $k<1$, the composed self-mapping $T^{q}: \bigcup_{i \in \bar{p}} A_{i} \rightarrow \bigcup_{i \in \bar{p}} A_{i}$ is contractive, and then continuous, and since $(X, d)$ is complete, since the associated $(X,\|\|)$ is a Banach space, $z \in \bigcap_{i \in \bar{p}} A_{i}$ is a unique fixed point of $T^{q}: \bigcup_{i \in \bar{p}} A_{i} \rightarrow \bigcup_{i \in \bar{p}} A_{i}$. Thus, again the continuity of $T^{q}: \bigcup_{i \in \bar{p}} A_{i} \rightarrow \bigcup_{i \in \bar{p}} A_{i}$ and the fact that it has a unique fixed point $z$ leads to the identities $T^{q}(T z)=T^{q+1} z=T\left(T^{q} z\right)=T z=T^{q}\left(T^{q} z\right)=T^{q} z$ so that $z=T z$ and then $z$ is also a fixed point of $T: \bigcup_{i \in \bar{p}} A_{i} \rightarrow \bigcup_{i \in \bar{p}} A_{i}$. Furthermore, $z$ is also the unique fixed point of $T: \bigcup_{i \in \bar{p}} A_{i} \rightarrow \bigcup_{i \in \bar{p}} A_{i}$ as follows by contradiction. Assume that $z$ is not unique. Then, such that $z$ is the unique fixed point of $T^{q}: \bigcup_{i \in \bar{p}} A_{i} \rightarrow \bigcup_{i \in \bar{p}} A_{i}$ and $\exists y(\neq z) \in \bigcap_{i \in \bar{p}} A_{i}$ such that $y$ and $z$ are both fixed points of $T: \bigcup_{i \in \bar{p}} A_{i} \rightarrow \bigcup_{i \in \bar{p}} A_{i}$. Then, $T^{q} y=T\left(T^{q} y\right)=T y=y$ which contradicts that $z$ is the unique fixed point of $T^{q}: \bigcup_{i \in \bar{p}} A_{i} \rightarrow \bigcup_{i \in \bar{p}} A_{i}$. Finally, as a result of the uniqueness of the fixed point, it follows directly that any $q$-tuple (3.30) converges to a unique $q$-tuple $\hat{z}:=(z, \ldots, z)=\hat{z}_{i}$; $\forall i \in \bar{p}$ for any $x \in \bigcup_{i \in \bar{p}} A_{i}$. Hence, Property (ii).

(B) $D \neq 0$ so that $A_{i} \cap A_{j} \neq \emptyset$ for $\forall i, j(\neq i) \in \bar{p}$. One can get from (3.6) to (3.8):

$$
\lim _{n \rightarrow \infty} d\left(T^{n q+j_{i}} x_{i}, T^{n q} x_{i}\right)=D, \limsup _{n \rightarrow \infty} d\left(T^{n q+\ell} x_{i}, T^{n q} x_{i}\right) \leq\left(\frac{\beta_{i} Q_{i}}{1-\alpha_{i} P_{i}}\right)^{\ell / s(x, x)} D,
$$

$; \forall x_{i} \in A_{i} ; \forall \ell \in \overline{j_{i}-1}, \forall i \in \bar{p}$. Thus:

$$
\lim _{n \rightarrow \infty} d\left(T^{(n+1) q+j_{i}} x_{i}, T^{n q} x_{i}\right)=D, \limsup _{n \rightarrow \infty} d\left(T^{(n+1) q+\ell} x_{i}, T^{n q} x_{i}\right) \leq\left(\frac{\beta_{i} Q_{i}}{1-\alpha_{i} P_{i}}\right)^{\ell / s(x, x)} D
$$

$; \forall x_{i} \in A_{i}, \forall \ell \in \overline{j_{i}-1}, \forall i \in \bar{p}$. One has from Lemma 3.3, Equation 3.24b and Proposition 3.2 of [14]:

$$
\lim _{m_{k}>\left(n_{k} \rightarrow \infty\right)} d\left(T^{m_{k} q+\sum_{\ell=i}^{m} j_{\ell}} x_{i}, T^{n_{k} q+\sum_{\ell=i}^{m^{\prime}} j_{\ell}} x_{i}\right)=d\left(\omega_{i+m+1}, \omega_{i+m^{\prime}+1}\right)=D
$$

, that is, the distance between the subsets $A_{i+m+1}\left(\equiv A_{i+m+1-p}\right.$ if $\left.m>p+1-i\right)$ and $A_{i+m^{\prime}+1}$ ( $\equiv A_{i+m^{\prime}+1-p}$ if $\left.m>m^{\prime}>p+1-i\right)$ of $X$ equalizes that of two corresponding best proximity points, for some convergent subsequences $T^{m_{k} q+\sum_{\ell=i}^{m} j_{\ell}} x_{i} \in A_{i+m+1}$ and $T^{n_{k} q+\sum_{\ell=i}^{m} j_{\ell}} x_{i} \in A_{i+m^{\prime}+1}$ and two best proximity points: $\omega_{i+m+1} \in A_{i+m+1} ; \omega_{i+m^{\prime}+1} \in A_{i}$ $+m^{\prime}+1$. Then, again from Lemma 3.3 and (3.34), one can get 


$$
\begin{aligned}
& \lim _{n \rightarrow \infty} d\left(T^{\left(n+n^{\prime}\right) q+\sum_{\ell=i}^{m} j_{\ell}} x_{i}, T^{n q+\sum_{\ell=i}^{m^{\prime}} j_{\ell}} x_{i}\right)=\lim _{n \rightarrow \infty} d\left(T^{\left(n+n^{\prime}\right) q+j_{i}} x_{i}, T^{n q} x_{i}\right) \\
& =d\left(\omega_{i+m+1}, \omega_{i+m^{\prime}+1}\right)=D
\end{aligned}
$$

Also, one has from Lemma 3.3, that $\lim _{n \rightarrow \infty} d\left(T^{n q+\sum_{\ell=i}^{m} j_{\ell}} x_{i}, T^{(n+1) q+\sum_{\ell=i}^{m} j_{\ell}} x_{i}\right)=0$ so that, by taking into account (3.35), $T^{n q+\sum_{\ell=i}^{m} j_{\ell}} x_{i} \rightarrow \omega_{i+m+1}\left(\in A_{i+m+1} \equiv A_{i+m+1-p}\right.$ if $\left.m>p+1-i\right)$ as $n \rightarrow \infty ; x \in A_{i}, \forall i, m(\geq i) \in \bar{p}$ with $j_{\ell}=j_{\ell-p}$ for any $\ell>p, \forall n^{\prime} \in N_{0}$, since $T^{n q+\sum_{\ell=i}^{m} j_{\ell}} x_{i} \in A_{i+m+1}$. That is, $T^{n q+\sum_{\ell=i}^{m} j_{\ell}} x_{i}$ converges to a best proximity point of $A_{i+m+1} ; \forall$ $x_{i} \in A_{i} ; \forall i \in \bar{p}$.

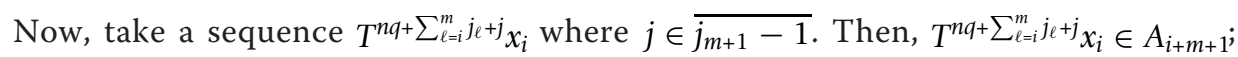
$\forall j \in \overline{j_{m+1}-1}$. Assume that $\left\{T^{n q+\sum_{\ell=i}^{m} j_{\ell+j}} x_{i}\right\}_{n \in N_{0}}$ does not converge in $A_{i+m+1}$ so that one can get from Theorem 3.1, Equation 3.8 and Lemma 3.3 (ii):

$$
\begin{aligned}
& 0<\liminf _{n \rightarrow \infty} d\left(T^{(n+1) q+\sum_{\ell=i}^{m} j_{\ell+j}} x_{i}, T^{n q+\sum_{\ell=i}^{m} j_{\ell}} x_{i}\right) \\
& \leq\left(\frac{\beta_{i} Q_{i}}{1-\alpha_{i} P_{i}}\right)^{j \ell / s(x, x)} \lim _{n \rightarrow \infty} d\left(T^{(n+1) q+\sum_{\ell=i}^{m} j_{\ell}} x_{i}, T^{n q+\sum_{\ell=i}^{m} j_{\ell}} x_{i}\right)=0 ; \\
& \forall x_{i} \in A_{i} ; \forall \ell \in \overline{j_{i}-1}, \forall j \in \overline{j_{m+1}-1}, \forall i \in \bar{p}
\end{aligned}
$$

which is a contradiction. Then, $\exists \lim _{n \rightarrow \infty} d\left(T^{(n+1) q+\sum_{\ell=i}^{m} j_{\ell}+j} x_{i}, T^{n q+\sum_{\ell=i}^{m} j_{\ell}} x_{i}\right)=0$ and $\left\{T^{n q+\sum_{\ell=i}^{m} j_{\ell+j}} x_{i}\right\}_{n \in N_{0}}$ is a Cauchy sequence with a limit in the closed and convex $A_{i+m+1}$ $\in X$ for any $j \in \overline{j_{m+1}-1}, \forall x_{i} \in A_{i}$ and $i \in \bar{p}$. It has been proven that $\hat{x}_{q n} \rightarrow \hat{z}_{i} ; \forall i \in \bar{p}$ and the set $\left\{\omega_{i}: i \in \bar{p}\right\}$ is a set of best proximity points with $\omega_{i+1}=T^{j_{i}} \omega_{i}=T^{j_{i}+q} \omega_{i} ; \forall i \in \bar{p}$ and $\omega_{p+1}=\omega_{1}$. It remains to prove that the elements of the limit sequence $\hat{z}_{i}$ are not dependent on the initial point $x_{i} \in A_{i} ; \forall i, m(\geq i) \in \bar{p}$ to construct any sequence of iterates except, perhaps, in the order that the limiting points are allocated within such a limiting sequence. Proceed by contradiction by assuming that there are two distinct best proximity points $\omega_{i}, z_{i} \in A_{i}$ for some $i \in \bar{p}$ so that:

$$
D=d\left(T^{j_{i}} \omega_{i}, \omega_{i}\right)<d\left(T^{j_{i}} \omega_{i}, z_{i}\right)=d\left(T^{j_{i}+n q} \omega_{i}, T^{n q} z_{i}\right)
$$

since $\omega_{i}, z_{i}\left(\neq \omega_{i}\right) \in A_{i}$ are best proximity points and $T^{j_{i}} \omega_{i} \in A_{i+1}$. Since that the above property holds irrespective of the integers $i, m(\geq i) \in \bar{p}$ and $n \in N_{0}$, the following contradiction follows from (3.37), and Theorem 3.1, Equation 3.6a:

$$
\begin{aligned}
& D=d\left(T^{j_{i}} \omega_{i}, \omega_{i}\right)=d\left(T^{j_{i}} z_{i}, z_{i}\right)<d\left(T^{j_{i}} \omega_{i}, z_{i}\right) \\
& =d\left(\omega_{i}, T^{j_{i}} z_{i}\right)=\lim _{n \rightarrow \infty} d\left(T^{j_{i}+n q} \omega_{i}, T^{n q} z_{i}\right)=D
\end{aligned}
$$

irrespective of $i \in \bar{p}$. Therefore, the best proximity points are unique within each of the subsets. Furthermore, since $T: \bigcup_{i \in \bar{p}} A_{i} \rightarrow \bigcup_{i \in \bar{p}} A_{i}$, the limit sequence (3.30) is unique by successive iterations from any of the best proximity points. Since there is a convergence to it from any initial point in $\bigcup_{i \in \bar{p}} A_{i}$, any $q$-sequence of iterates converges to such a limit sequence, irrespective of the initial point, except for the order of the elements.

Remark 3.5. Concerning with Theorem 3.4 (ii), note that $T: \bigcup_{i \in \bar{p}} A_{i} \rightarrow \bigcup_{i \in \bar{p}} A_{i}$ is not necessarily contractive when $D=0$ although $T^{q}: \bigcup_{i \in \bar{p}} A_{i} \rightarrow \bigcup_{i \in \bar{p}} A_{i}$ be contractive. 
However, the contractive property of $T^{q}: \bigcup_{i \in \bar{p}} A_{i} \rightarrow \bigcup_{i \in \bar{p}} A_{i}$ leads to that of $T: \bigcup_{i \in \bar{p}} A_{i} \rightarrow \bigcup_{i \in \bar{p}} A_{i}$ possessing also a unique fixed point in $\bigcap_{i \in \bar{p}} A_{i}$ which is that of $T^{q}: \bigcup_{i \in \bar{p}} A_{i} \rightarrow \bigcup_{i \in \bar{p}} A_{i}$. It is also noted that the limit sequence is unique except in the order of the elements in the sense that if a sequence of iterates $\hat{x}_{q n}$ Equation 3.31 converges to $\hat{z}_{i}$ Equation 3.30 for any initial point $x \in A_{i}$, then for $x \in A_{k}$ the limit sequence being asymptotically reached will be:

$$
\begin{aligned}
& \hat{z}_{k}:=\left(T z_{k}=T^{q+1} z_{k}, \ldots, \omega_{j+1}=T^{j_{k}} z_{k}, T^{j_{k}+1} z_{k}, \ldots, \omega_{k+2}=T^{j_{k}+j_{k+1}} z_{i},\right. \\
& \left.T^{j_{k}+j_{k+1}+1} z_{k}, \ldots, \omega_{k+p}=\omega_{k}=z_{k}=T^{q} z_{k}\right)
\end{aligned}
$$

which is identical to (3.30) except in the order of its elements. $\square$

An example is given below

Example 3.6: Take $p=2$ and subsets $A_{1} \equiv C\left(a, 0, a-a_{0}\right)$ and $A_{2} \equiv C\left(-a, 0, a-a_{0}\right)$ of $\boldsymbol{R}^{2}$ are circles of centre in $(a, 0)$ and $(-a, 0)$, respectively, and radius $a$ - $a_{0}$ with $a>a_{0}$ which are defined by

$$
\begin{aligned}
& A_{1}:=\left\{(x, y) \in R^{2}:(x-a)^{2}+y^{2} \leq\left(a-a_{0}\right)^{2}\right\} \\
& A_{2}:=\left\{(x, y) \in R^{2}:(x+a)^{2}+y^{2} \leq\left(a-a_{0}\right)^{2}\right\}
\end{aligned}
$$

We consider the complete metric space $\left(\boldsymbol{R}^{2}, d\right)$ with the Euclidean metric. It is clear that such a space being considered as the Banach space $\left(\boldsymbol{R}^{2},\|\|\right)$, endowed with the Euclidean norm, is uniformly convex, then strictly convex and reflexive [23]. It is noted that $\mathrm{D}=2 a_{0}=\operatorname{dist}\left(A_{1}, A_{2}\right)$ and now consider the constraint (3.1) with functions $s, x, r, t:\left(A_{1} \cup A_{2}\right) \times\left(A_{1} \cup A_{2}\right) \rightarrow \boldsymbol{R}_{+}$being constant identically unity. Assume that $T$ is some extended 2-cyclic self-mapping on $A_{1} \cup A_{2}$ with $j_{1}=2, j_{2}=1$. Thus, one can get

$$
\begin{aligned}
& g=(x, y) \in A_{1} \Rightarrow T g \in A_{1}, T^{2} g \in A_{2}, T^{3} g \in A_{1}, T^{4} g \in A_{1}, T^{5} g \in A_{2}, \\
& g=(x, y) \in A_{2} \Rightarrow T g \in A_{1}, T^{2} g \in A_{1}, T^{3} g \in A_{2}, T^{4} g \in A_{1}, T^{5} g \in A_{1},
\end{aligned}
$$

Now, define also the family of parameterized circles $\hat{A}_{a_{1}}:=\left\{(x, y) \in R^{2}:(x-a)^{2}+y^{2} \leq a_{1}^{2}\right\} \subseteq A_{1}$ being with $A_{1}$ and contained in it (with proper or improper set inclusion) of radius $0<a_{1} \leq a-a^{\prime}$ for $a^{\prime} \in[0, a)$. It is noted that $\hat{A}_{a} \equiv A_{1}$. Next, we define constructively a self-mapping which is an extended cyclic one and which verifies the properties. Now, consider a self-mapping $T: A_{1} \cup A_{2} \rightarrow A_{1} \cup A_{2}$ defined as follows:

$$
\begin{aligned}
& g_{0}=\left(x_{0}, y_{0}\right) \in A_{1} ; \\
& g_{1}=\left(x_{1}, y_{1}\right)=T g_{0}=\left(\hat{k}_{1}\left(x_{0}, y_{0}\right) x_{0}, \hat{k}_{1}\left(x_{0}, y_{0}\right) y_{0}\right)\left(\in F r \hat{A}_{a_{1}} \subseteq A_{1}\right) \\
& =\left\{(x, y):\left(\hat{k}_{1} x_{0}-a\right)^{2}+\hat{k}_{1}^{2} y_{0}^{2}=a_{1}^{2} \leq\left(a-a^{\prime}\right)^{2}\right\}
\end{aligned}
$$

The positive solution in $\hat{k}_{1}$ of the equality defining a circle $\hat{A}_{a_{1}}$ for a fixed $a_{1}=a_{1}\left(x_{0}\right.$, $\left.y_{0}\right) \leq a-a^{\prime}$ is defined below together with available point-dependent lower and upperbounds: 


$$
\frac{a}{2 a+a_{0}} \leq \frac{a x_{0}}{x_{0}^{2}+y_{0}^{2}} \leq \hat{k}_{1}=\hat{k}_{1}\left(x_{0}, y_{0}\right)=\frac{2 a x_{0}+\sqrt{4 a^{2} x_{0}^{2}-4\left(a^{2}-a_{1}^{2}\right)\left(x_{0}^{2}+y_{0}^{2}\right)}}{2\left(x_{0}^{2}+y_{0}^{2}\right)} \leq \frac{2 a x_{0}}{x_{0}^{2}+y_{0}^{2}} \leq \frac{a}{x_{0}} \leq \bar{k}_{1}:=\frac{a}{a_{0}}
$$

It is noted that such a positive solution always exists everywhere in $A_{1}$ since $a \geq a_{1} \geq a \sqrt{1-\frac{x_{0}^{2}}{x_{0}^{2}+y_{0}^{2}}}$. Thus, the constraint (3.1) is fulfilled by any self-mapping $T$ : $A_{1} \cup A_{2} \rightarrow A_{1} \cup A_{2}$ with $1>\alpha_{1} \geq 0, \quad \beta_{1}=\frac{a}{a_{0}}\left(1-\alpha_{1}\right)>1-\alpha_{1}$ and $k_{1}:=\bar{k}_{1}^{2}=\left(\frac{a}{a_{0}}\right)^{2}=\left(\frac{\beta_{1}}{1-\alpha_{1}}\right)^{2}>1$. It is noted that the condition (3.1) is not guaranteed to be contractive for any point of $A_{1}$. It is also noted that if $\left(x_{0}, y_{0}\right) \in \operatorname{Fr}\left(A_{1}\right)$ then $\hat{k}_{1}\left(x_{0}, y_{0}\right)=1$ with $a_{0}=0$ so that $g_{1}=T g_{0}=g_{0}$. However, it can be noticed that $g_{1}$ is not a fixed point since $g_{2}=T g_{1} \neq g_{1}$. Next, define:

$$
\begin{aligned}
& g_{2}=\left(x_{2}, y_{2}\right)=T g_{1}=T^{2} g_{0}=\left(\min \left(-\hat{k}_{1} x_{1},-\hat{k}_{1} a_{0}\right),-\hat{k}_{1} y_{1}\right)\left(\in A_{2}\right) \\
& \Rightarrow\left[\left(x_{1}=a_{0}, y_{1}=0\right) \Rightarrow\left(\hat{k}_{1}=1, x_{2}=-a_{0}, y_{2}=0\right)\right] \Rightarrow d\left(g_{1}, g_{2}\right)=D \\
& g_{3}=\left(x_{3}, y_{3}\right)=T g_{2}=T^{2} g_{1}=T^{3} g_{0}=\left(\max \left(-k_{2} x_{2},-k_{2} a_{0}\right),-k_{2} y_{2}\right)\left(\in A_{1}\right) \\
& \Rightarrow\left[\left(x_{2}=-a_{0}, y_{2}=0\right) \Rightarrow\left(x_{3}=a_{0}, y_{3}=0\right)\right] \Rightarrow d\left(g_{3}, g_{2}\right)=D
\end{aligned}
$$

under the contractive constant $k_{2}$ defined as follows subject to constraints:

$$
k_{2}:=\frac{\beta_{2}}{1-\alpha_{2}}<k_{1}^{-1}=\left(\frac{a_{0}}{a}\right)^{2}=\left(\frac{1-\alpha_{1}}{\beta_{1}}\right)^{2}
$$

so that the composed extended cyclic self-mapping $T^{3}: A_{1} \cup A_{2} \rightarrow A_{1} \cup A_{2}$ is subject to a the contractive condition (3.1) of contractive constant $k=k_{1} k_{2}<1$ with $s, x, r, t$ : $\left(A_{1} \cup A_{2}\right) \times\left(A_{1} \cup A_{2}\right) \rightarrow \boldsymbol{R}_{+}$, i.e. for the Euclidean distance. Consider initial points in $A_{2}$ as $g_{0}=\left(x_{0}, y_{0}\right) \in A_{2}$. Thus, (a) first apply (3.45) with the replacements $\left(x_{3}, y_{3}\right) \rightarrow$ $\left(x_{1}, y_{1}\right) \in A_{2}$ and $g_{3} \rightarrow g_{1}\left(x_{1}, y_{1}\right) \in A_{1}$; (b) then apply (3.42) with the replacement ( $x_{3}$, $\left.y_{3}\right) \rightarrow\left(x_{1}, y_{1}\right)$ and $g_{1} \rightarrow g_{2}=\left(x_{2}, y_{2}\right) \in A_{1}$; (c) later on apply (3.44) with the replacement $\left(x_{2}, y_{2}\right) \rightarrow\left(x_{3}, y_{3}\right)$ and $g_{2} \rightarrow g_{3}\left(x_{3}, y_{3}\right) \in A_{2}$. Theorems 3.1 and 3.4 are fulfilled and there is a limiting repeated sequence of three points $\left.S^{*}:=\left(\left(-a_{0}, 0\right)\right),\left(a_{0}, 0\right),\left(a_{0}, 0\right)\right)$ which are two best proximity points, one located in $A_{2}$ and another one in $A_{1}$, repeated. It is noted that the repeated value of the best proximity point $\left(a_{0}, 0\right)$ in the limiting sequence is due to the fact that the circumference being the boundary of $A_{1}$ is invariant under $T$, although infinitely many different cyclic self-mappings can be defined on the same two subsets $A_{1,2}$ so that a limiting sequence is reached having a common (perhaps only) the best proximity points with $S^{*}$. It is also noted that if $a_{0} \neq$ 0 then $\left(a_{0}, 0\right)$ is not a fixed point of $T: A_{1} \cup A_{2} \rightarrow A_{1} \cup A_{2}$ since $T\left(a_{0}, 0\right)=\left(a_{0}, 0\right) \Rightarrow$ $T^{2}\left(a_{0}, 0\right)=\left(-a_{0}, 0\right)$. However, if $a_{0}=D=0$ then the intersection of both circles is $\{(0$, $0)\}$ so that the limiting sequence consists of a repeated fixed point.

\section{Acknowledgements}

The authors wish to thank the Spanish Ministry of Education for their support through Grant DPI2009-07197. They are also grateful to the Basque Government for their support through Grants IT378-10, and SAIOTEK SPE09UN12. The authors thank the referees for their useful suggestions. 


\section{Author details}

'Instituto de Investigacion y Desarrollo de Procesos, Universidad del Pais Vasco, Campus of Leioa (Bizkaia), Aptdo. 644Bilbao, 48080-Bilbao, Spain ²Department of Mathematics, Texas A\&M University- Kingsville, 700 University Blvd., Kingsville, TX 78363-8202, USA

\section{Authors' contributions}

Both the authors contributed equally and significantly in writing this paper. All authors read and approved the final manuscript

\section{Competing interests}

The authors declare that they have no competing interests.

Received: 16 May 2011 Accepted: 28 September 2011 Published: 28 September 2011

\section{References}

1. Harjani, J, Lopez, B, Sadarangani, K: A fixed point theorem for mappings satisfying a contractive condition of rational type of partially ordered metric space. Abstr Appl Anal 2010, 8 (2010). (Article Number 190701)

2. Harjani, J, Sadarangani, K: Fixed point theorems for weakly contractive mappings in partly ordered sets. Nonlinear Anal Theory Methods Appl. 71(7-8), 3403-3410 (2009). doi:10.1016/.na.2009.01.240

3. Bhardwaj, R, Rajput, SS, Yadava, RN: Application of fixed point theory in metric spaces. Thai J Math. 5(2), 253-259 (2007)

4. Enjouji, Y, Nakanishi, M, Suzuki, T: A generalization of Kannan's fixed point theorem. Fixed Point Theory Appl 2009, 10 (2009). (Article Number 192872)

5. Banach, S: Sur les operations dans les ensembles abstracts et leur application aux équations intégrales. Fundamenta Mathematicae. 3, 133181 (1922)

6. Chatterjee, SK: Fixed point theorems. Comptes Ren Acad Bulgaria Sci. 25, 727730 (1972)

7. Fisher, B: A fixed point theorem for compact metric spaces. Publ Math Debrecen. 25, 193194 (1978)

8. Kannan, R: Some results on fixed points. Bull Calcutta Math Soc. 60, 7176 (1968)

9. Kannan, R: Some results on fixed points-II. Am Math Monthly. 76, 405408 (1969)

10. Reich, S: Some remarks concerning contraction mappings. Can Math Bull. 14, 121124 (1971)

11. Kikkawa, M, Suzuki, T: Some similarity between contractions and Kannan mappings. Fixed Point Theory Appl 2008, 8 (2008). (Article ID 649749)

12. Subrahmanyam, PV: Completeness and fixed points. Monatshefte für Mathematik. 80(4), 325-330 (1975). doi:10.1007/ BF01472580

13. Kirk, WA, Srinivasan, PS, Veeramani, P: Fixed points for mappings satisfying cyclical contractive conditions. Fixed Point Theory. 4(1), 79-89 (2003)

14. Eldred, AA, Veeramani, P: Existence and convergence of best proximity points. J Math Anal Appl. 323(2), 1001-1006 (2006). doi:10.1016/j.jmaa.2005.10.08

15. Karpagam, S, Agrawal, S: Best proximity point theorems for p-cyclic Meir-Keeler contractions. Fixed Point Theory Appl 2009, 9 (2009). (Article Number 197308)

16. Di Bari, S, Suzuki, T, Vetro, C: Best proximity points for cyclic Meir-Keeler contractions. Nonlinear Anal.: Theory Methods Appl. 69(11), 3790-3794 (2008). doi:10.1016/j.na.2007.10.014

17. De la Sen, M: Linking contractive self-mappings and cyclic Meir-Keeler contractions with Kannan self-mappings. Fixed Point Theory Appl 2010, 23 (2010). (Article Number 572057)

18. De la Sen, M: Some combined relations between contractive mappings, Kannan mappings reasonable expansive mappings and T-stability. Fixed Point Theory Appl 2009, 25 (2009). (Article Number 815637)

19. Kikkawa, M, Suzuki, T: Three fixed point theorems for generalized contractions with constants in complete metric spaces. Nonlinear Anal: Theory Methods Appl. 69(9), 2942-2949 (2008). doi:10.1016/j.na.2007.08.064

20. Suzuki, T: Some notes on Meir-Keeler contractions and L-functions. Bull Kyushu Inst Technol. 53, 1213 (2006)

21. Derafshpour, M, Rezapour, S, Shahzad, N: On the existence of best proximity points of cyclic contractions. Adv Dyn Syst Appl. 6(1), 33-40 (2011)

22. Rezapour, Sh, Derafshpour, M, Shahzad, N: Best proximity points of cyclic $\varphi$-contractions on reflexive Banach spaces. Fixed Point Theory Appl 2010, 7 (2010). (Article ID 946178)

23. Qin, X, Kang, SM, Agarwal, RP: On the convergence of an implicit iterative process for generalized asymptotically quasi non-expansive mappings. Fixed Point Theory Appl 2010, 19 (2010). (Article Number 714860)

24. Rus, IA: Cyclic representations and fixed points. Ann T Popoviciu Seminar Funct Eq Approx Convexity. 3, 171178 (2005)

25. Pacurar, M, Rus, IA: Fixed point theory for cyclic $\varphi$-contraction. Nonlinear Anal: Theory Methods Appl. 72(3-4), 1181-1187 (2010). doi:10.1016/i.na.2009.08.002

26. Karapinar, E: Fixed point theory for cyclic weak $\varphi$-contraction. Appl Math Lett. 24, 822825 (2011)

27. Shazhad, N, Sadiq Basha, S, Jeyaraj, R: Common best proximity points: global optimal solutions. J Optim Theory Appl. 148(1), 69-78 (2011). doi:10.1007/s10957-010-9745-7

28. Al-Thagafi, MA, Shahzad, N: Convergence and existence results for best proximity points. Nonlinear Anal: Theory Methods Appl. 70(10), 3665-3671 (2009). doi:10.1016/..na.2008.07.022

29. Vetro, C: Best proximity points: convergence and existence theorems for p-cyclic mappings. Nonlinear Anal: Theory Methods Appl. 73(7), 2283-2291 (2010). doi:10.1016/..na.2010.06.008

doi:10.1186/1687-1812-2011-59

Cite this article as: De la Sen and Agarwal: Some fixed point-type results for a class of extended cyclic selfmappings with a more general contractive condition. Fixed Point Theory and Applications 2011 2011:59. 